\title{
Teledermatology in Nepal: Where are we?
}

\author{
Neupane Saraswoti S \\ Lecturer, \\ Department of Dermatology and Venereology \\ Nepalgunj Medical College Teaching Hospital, \\ Kohalpur, Banke, Nepal \\ E-mail: sarunpn@yahoo.com
}

\begin{abstract}
Teledermatology is the practice of clinical dermatology by making use of images, computers and telecommunication technologies for the diagnosis and treatment of skin conditions. The technology involves two different approaches, store-and-forward approach and real-time interaction. Although it has a long history, its implementation in Nepal is still in the period of conception. Both the approaches have their own advantages, but they are not free from disadvantages. In Nepal, rapidly growing telecommunication facility throughout the country can be utilized to increase the accessibility to dermatology consultation service. Considering the high burden of skin diseases compared to the limited number of dermatologists in the country, teledermatology could be an effective tool in improvement of the health care delivery system.

Keywords: Teledermatology, skin diseases, Nepal.
\end{abstract}

\section{Introduction}

The use of computers and telecommunication technology to facilitate the provision of health care for persons with skin disease is an area of increasing interest and activity. Telemedicine is defined by the World Health Organization (WHO) as 'the practice of healthcare using interactive audio, visual and data communications. This includes healthcare delivery, diagnoses, consultation and treatment as well as education and transfer of medical data'. Telemedicine was first used by a psychiatrist in USA using black and white close circuit TV video monitors. ${ }^{1}$ Since then, telemedicine, including teledermatology has been implemented in different countries throughout the world. Telemedicine is widely practiced in different speciality of medicine including dermatology. The term 'teledermatology' refers to the practice of clinical dermatology by telemedicine, that is, by making use of images, computers and telecommunication technologies for the diagnosis and treatment of skin conditions. In this, patient from the remote areas can access the dermatology consultant service. So, this is a dermatology consultation service at a distance. Although the gold standard for any specialist referral remains the traditional face-to-face consultation, teledermatology offers improved access to specialist care for rural and disabled patients with skin diseases.

In Nepal, telecommunication facility and internet technology is advancing rapidly and the goal is to provide the facility throughout the country. Here, skin diseases is ranked first among the top ten diseases accounting for morbidity and is the commonest reason for accessing healthcare services. ${ }^{2}$ Eighty four percent of households are concentrated in the rural areas. The number of dermatologists is few, approximately one for every half a million population and the few dermatologists are also concentrated in the urban areas. In this context where there are only few dermatologists compared to the high burden of skin diseases and even those few dermatologists are concentrated in the urban areas, teledermatology may be utilized to bridge the gap. In the rural areas patients have to travel a long distance to get the specialist care which adds to the financial burden and is time consuming as well. Teledermatology offers an obvious method of service delivery directed towards improving access and decreasing cost, by eliminating travel for patients as well as physicians. Traditionally, doctor/patient relationship has long been regarded as the 'gold standard' in the health care delivery that is why some authors consider that the use of teledermatology might erode the doctor/patient relationship., ${ }^{3,4}$ But in Nepal considering the difficulties and the burden of skin diseases teledermatology can be taken as a positive change in the healthcare delivery system. The first workshop in teledermatology was held in November 2005 at B.P. Koirala Institute of Health Sciences, Dharan, where dermatologists from different parts of the country had actively participated. Although initiation has already been taken to start Teledermatology, it is still in the period of conception.

\section{Teledermatology technology}

Consultations may be "Real-time" using videoconferencing equipment or "store-and-forward" 
using prerecorded text and images.

Store-and-forward technology (SAF) In a store-and-forward system, the referring doctor enters the clinical information and digital still images on to a computer. This is transmitted directly via a modem or the internet to a central computer through which the dermatologist may then access the data independently, at a convenient time and send the diagnosis, treatment and advices via internet. Hence, this is an indirect method of consultation.

\section{Real-time technology (R'T)}

Real-time technology allows direct interaction between the dermatologist, the patient and the person conducting the video imaging. The video conferencing equipment enables the dermatologist to see the patient through the video link, while the patient has contact with the dermatologist through a small digital camera mounted on the videoconferencing unit. While store-and-forward technology uses still images, RT technology uses moving images taken by a video camera. High band width internet facility is required in this technique, although lower quality transmissions are possible over telephone lines. With some exceptions, store-and-forward technology has been favored in the US, whereas real-time interactive techniques have predominated in Europe and Australia.

\section{Diagnostic Reliability and Accuracy} To be effective and safe, the teledermatology process needs to demonstrate acceptable levels of accuracy and reliability. Accuracy is reflected by the degree of concordance between the teledermatology and face-to-face diagnoses. Reliability is dependent on how consistently a set of results can be reproduced across different operators. Different studies have been conducted to find out the diagnostic reliability and accuracy. A study conducted by Lim AC and colleagues ${ }^{5}$ have demonstrated high reliability and accuracy in diagnosis with mean concordance rate achieved by four dermatologists studying 53 store-andforward diagnostic cases, originating from 49 referred patients, was $79 \%$ when primary diagnosis was considered that narrowed further to $86 \%$ when differential diagnoses was considered. Study conducted by Tait $\mathrm{CP}^{6}$ demonstrated diagnostic accuracy of $83 \%$ using store-and-forward technology which was comparable to that obtained by other studies using real-time technology in New Zealand ${ }^{7}$ and Northern Ireland. ${ }^{8}$ Using storeand-forward technology, one study found that the teledermatologists provided a correct diagnosis for $90 \%$ of the patients. ${ }^{9}$

The existing literature suggests that teledermatology, whether using store-and-forward or real-time interactive technology, performs comparably well with the level of agreement expected between two different clinic-based examiners. ${ }^{10}$ Lesher JL and colleagues found $94 \%$ agreement between clinic-based examiners. ${ }^{11}$ Agreement between clinic-based dermatologists and teledermatologists, using a real-time interactive technology, was also high in this study that is $78 \%$. A study ${ }^{12}$ using store-and-forward technology employed multiple clinic-based dermatologists and teledermatologists, which allowed for reliability assessments to be made on the same patient sample, found that the rate of agreement between pairs was comparable to that found between two clinic-based examiners.

\section{Cost effectiveness}

Earlier studies showed that RT teledermatology was more clinically efficient than the SAF variant. ${ }^{13}$ With the ever-increasing cost of health care provision, some have looked to teledermatology to reduce waiting lists and costs. ${ }^{14}$ Studies ${ }^{15,16,17}$ comparing the cost-effectiveness of real-time teledermatology with conventional hospital outpatient appointments showed advantages for the patients in terms of time off work, loss of income to the patient or productivity by the employer as well as time and expense of traveling to hospital. Real-time teledermatology was less advantageous to the health care system, being more expensive and time-consuming for the General Practitioner and dermatologist. In the opinion of several authors, ${ }^{18,19}$ store-and-forward teledermatology could be more cost-effective than real-time consultation due to its speed and convenience. A study conducted by loane MA and colleagues to assess the cost benefit analysis of Teledermatology have concluded that the storeand-forward photographic consultation was much more economical, but less clinically effective, than real-time videoconferencing consultation because, in the latter case, the dermatologist could extract clinically useful information through talking to the patient. ${ }^{16}$ However, different recent review studies ${ }^{23,24}$ have pointed on the lack of economic evaluation of SAF teledermatology.

\section{Satisfaction}

Each of 3 different groups must find teledermatology acceptable and satisfactory - the referring clinician, the teledermatologist, and the patient. In some cases, patients have felt uncomfortable (18\% of respondents) and embarrassed (17\% of respondents) when using a real-time consult link. ${ }^{20}$ In a study assessing referring clinicians' satisfaction, $98 \%$ of the respondents believed the real-time teledermatology consult system provided a valuable experience and $75 \%$ believed they received an educational benefit. ${ }^{20}$ Another study reported that referring 
clinicians were satisfied with the teledermatology consult system but found that the time commitment needed as a telepresenter for the real-time consults was burdensome. ${ }^{21}$ Some interesting insights arose from a survey of referring clinicians' perceptions of store-and-forward teledermatology prior to its introduction into their practices. ${ }^{22}$ Although only $15 \%$ of respondents had high expectations for teledermatology, most were unsure $(81 \%)$. Likewise, only $27 \%$ expressed confidence in diagnosis and management through teledermatology and most were unsure (69\%). Despite some uncertainty about the system, the majority of respondents were enthusiastic about being part of teledermatology studies $(70 \%)$.

\section{Pros and cons}

Both the store-and-forward approaches as well as the real-time interactive process have its own advantage and disadvantages. Considering the advantages of the store-and-forward approach, it is inexpensive, can be used as a triage tool, dermatologists can review large numbers of images to increase efficiency and it can reduce the time from referral to diagnosis. Regarding its disadvantages, as there is no direct conversation between the patient and the dermatologist, there may be information gaps that can lead to difficulties in making diagnosis. A rapport may not be developed between patient and the teledermatologist and psychological issues cannot be addressed. Legal and security. issues exist regarding the identity of the person being photographed.Real-time interaction technology, as it includes direct interaction, it allows collection of all required clinical information. It can also provide an opportunity for third-party education. Costs continue to decrease because of advancing internet technology and usage. Disadvantages are: it is as time consuming for doctors as face-to-face consultations. Picture quality may be less than optimal. The patient, dermatologist, and the referring physician must be available at the same time. It is expensive in terms of capital and running cost.

\section{Conclusion}

With the invent of computer, video camera, digital camera and advancing internet technology, telerdermatology is possible even in Nepal, where patient from the remote places need to travel a long distance for dermatology consultation, teledermatology may be an effective tool. Primary care physician may contact dermatologist through Teledermatology for diagnosis and management or even one dermatologist may contact the other for second opinion whenever needed. So, the government may take an initiative to establish Teledermatology projects to make healthcare delivery more effective so that dermatology service may be accessible to each corner throughout the country.
References:

1. Berdick AE, Burman B. Teledermatology. Adv. Dermatol 1997; 12: 19-45.

2. Government of Nepal.Ministry of Health and Population.Fact sheets.Top ten diseases accounting to morbidity http://www moh.gov.np/ Home/FACT.ASP\# Disease (accessed $18^{\text {th }}$ October 2008).

3. Wootton R, Darkins A. Telemedicine and the doctorpatient relationship. J R Coll Phys London 1997; 31: 598-9.

4. Perednia DA. Fear, loathing, dermatology, and telemedicine. Arch Dermatol 1997; 133: 151-5.

5. Lim AC, Egerton IB, See A and Shumack SP. Accuracy and reliability of store-and-forward teledermatology: Preliminary results from the St George Teledermatology Project. Australas. J.Dermatol. 2001; 42: 247-251

6. Tait CP and Clay CD. Pilot study of store-and-forward teledermatology services in Perth, Western Australia Australas. J.Dermatol 1999; 40: 190-193.

7. Oakley AM, Astwood DR, Loane M, Duffill MB, Rademaker M, Wootton R. Diagnostic accuracy of teledermatology: Results of a preliminary study in New Zealand. N.Z. Med. J. 1997; 110: 51-3.

8. Loane MA, Gore HE, Corbett R. Preliminary results from the Northern Ireland arms of the UK multicentre teledermatology trial: Effect of camera performance on diagnostic accuracy. J. Telemed. Telecare 1997; 3: S73-5.

9. Zelickson BD, Homan L. Teledermatology in the nursing home. Arch Dermatol 1997; 133: 171-4.

10. Whited JD. Teledermatology Current Status and Future Directions. Am J Clin Dermatol 2001; 2 (2): 59-64.

11. Lesher JL, Davis LS, Gourdin FW. Telemedicine evaluation of cutaneous diseases: a blinded comparative study. J Am Acad Dermatol 1998; 38: 27-31

12. Hall RP, Simel DL. Reliability and accuracy of dermatologists' clinic-based and digital image consultations. J Am Acad Dermatol 1999; 41: 693-702

13. Loane MA, Bloomer SE, Corbett R. A comparison of real-time and store-and-forward teledermatology: a costbenefit study. Br J Dermatol 2000; 143:1241-7.

14. Eedy DJ, Wootton R. Teledermatology: a review. Br J Dermatol 2001; 144: 696-707

15. Oakley AM, Kerr P, Dunfill M. Patient cost-benefits of realtime teledermatology - a comparision of data from Northern Ireland and New Zealand. J Telemed Telecare 2000; 6: 97-101.

16. Loane MA, Bloomer SE, Corbett R. Patient cost-benefit analysis of teledermatology measured in a randomised control trial. J Telemed Telecare 1999; 5 (Suppl. 1): S1-3.

17. Wootton R, Bloomer SE, Corbett R. Multicentre randomized control trial comparing real-time teledermatology with conventional outpatient dermatological care: a societal cost-benefit analysis. $\mathrm{Br}$ Med J 2000; 320: 1252-6.

18. D'Souza M, Shah D, Misch K, Ostlere L. Dermatology opinions via intranet could reduce waiting times. Br Med J 1999; 318: 737 (Letter.).

19. Harrison PV, Kirby B, Dickinson Y, Schofield R. Teledermatology high technology or not? J Telemed Telecare 1998; 4: 31-2.

20. Gilmour E, Campbell SM, Loane MA, et al. Comparison of teleconsultations and face-to-face consultations: preliminary results of a United Kingdom multicentre teledermatology study. Br J Dermatol 1998; 139: 81-7

21. Jones DH, Crichton C, Macdonald A. Teledermatology Scotland. J Telemed Telecare 1996; 2 Suppl. 1: 7-9

22. Collins K, Nicolson P, Bowns I. General practitioners' perceptions of storeand- forward teledermatology. $\mathrm{J}$ Telemed Telecare 2000; 6: 50-3

23. Whited JD. Teledermatology research review. Int J Dermatol 2006; 45:220-9.

24. Eminovic N, Keizer NF, Bindels PJ, Hasman A. Maturity of teledermatology evaluation research: a systematic literature review Br J Dermatol. 2007; 156:412-419. 